\title{
ESTRATEGIAS DE AFRONTAMIENTO EN UNA MUESTRA DE ESTUDIANTES UNIVERSITARIOS
}

\author{
Francisco Manuel Morales Rodríguez \\ Facultad de Psicología. Universidad de Granada \\ fmmorales@ugr.es
}

Fecha de Recepción: 4 Abril 2018

Fecha de Admisión: 10 Abril 2018

\section{RESUMEN}

Introducción: La evaluación de las estrategias de afrontamiento empleadas por los estudiantes universitarios se considera fundamental ya que tiene implicaciones relevantes desde el punto de vista educativo y clínico ya que el empleo de estrategias funcionales o productivas guarda relación con mayor bienestar, calidad de vida, adaptación 0 ajuste en el ámbito universitario. Ello permitirá generar toma de conciencia sobre las estrategias empleadas por el estudiantado para que por parte del profesorado se desarrollen programas para el entrenamiento de aquellas consideradas más productivas ante los continuos retos y desafíos que se vienen planteando desde la Educación Superior. Objetivos: Examinar las estrategias de afrontamiento empleadas por una muestra de estudiantes universitarios españoles. Método: Los participantes fueron 169 estudiantes de grado pertenecientes a la Facultad de Ciencias de la Educación. Para evaluar las estrategias de afrontamiento se aplicó la forma general de una escala validada en población española.

Resultados: Este estudio presenta algunos de los datos preliminares obtenidos en esta investigación, analizados y discutidos según la literatura científica revisada, en la que se pone de manifiesto las estrategias de afrontamiento más empleadas por esta muestra de estudiantes universitarios, entre las que se encuentra tanto el empleo de estrategias de afrontamiento centradas en la solución del problema, así como aquellas otras centradas en la emoción. Conclusiones: Los datos obtenidos son relevantes para generar toma de conciencia de las estrategias de afrontamiento empleadas en el ámbito universitario para el diseño de un futuro programa o intervención psicoeducativa que permita entrenar aquellas estrategias de afrontamiento que resulten más eficaces para el afrontamiento de situaciones académicas generadoras de estrés.

Palabras clave: estrategias de afrontamiento; evaluación; Educación Superior; intervención psicoeducativa

\section{ABSTRACT}

Coping strategies in a sample of university students.

Introduction: The evaluation of coping strategies used by university students is considered fun- 


\section{ESTRATEGIAS DE AFRONTAMIENTO EN UNA MUESTRA DE ESTUDIANTES UNIVERSITARIOS}

damental due to it's relevant educational and clinical impact, since the use of functional or productive strategies is related to greater well-being, quality of life and adaptation or adjustment within the university environment. This will make it possible to generate awareness of the strategies employed by the student body. Then in the face of the continuous challenges and challenges that arise from Higher Education teachers can develop programs for training students in those considered most productive. Objectives: To examine the coping strategies employed by a sample of Spanish university students. Method: The participants were 169 undergraduates belonging to the Faculty of Educational Sciences. The way used to evaluate the coping strategies was the general scale approved in the Spanish population. Results: This study displays some of the preliminary data obtained in the research. These have been analysed and discussed according to reviewed scientific literature, and they highlight the coping strategies most used by this sample of university students. Among the coping strategies used are found both those which focus on solving the problem, and those which focus on emotions. Conclusions: The data obtained is relevant to generate awareness of the coping strategies used within the university environment for the design of a future program or psychoeducational initiatives. These will provide training in the more effective coping strategies in order to deal with academic situations that generate stress.

Keywords: coping strategies; evaluation; Higher education; psychoeducational initiatives

\section{ANTECEDENTES}

Las estrategias de afrontamiento se definen como el proceso dinámico de intentar reducir o eliminar los estresores a través de respuestas cognitivas o de comportamiento (Lazarus y Folkman, 1984). De acuerdo con un modelo transaccional de afrontamiento (Lazarus, 1991), la valoración primaria de un evento o situación como amenazante, dañino o desafiante, junto con su valoración secundaria de si tienen o no los recursos para hacer frente al evento estresante, influye en el tipo de estrategia de afrontamiento que se implementa.

Se espera que el afrontamiento modere y medie las asociaciones entre el estrés y los problemas de salud mental (Compas, Connor-Smith, Saltzman, Thomsen y Wadsworth, 2001). Connor-Smith y Compas (2002) encontraron que varias estrategias de afrontamiento (por ejemplo, el compromiso de control primario, el compromiso de control secundario y el afrontamiento de la separación) moderaron las relaciones entre la reactividad al estrés involuntaria, el estado de salud y los problemas de internalización. Las reacciones efectivas / ajustadas fueron aquellas reacciones, que proporcionaron un efecto de amortiguación, mientras que las reacciones de afrontamiento ineficaces / inadaptadas ponen al niño en riesgo de desarrollar problemas mentales. Compas et al. (2001) revisaron los estudios empíricos de la asociación entre el afrontamiento y los resultados psicológicos. Se pone de manifiesto que el afrontamiento centrado en el problema puede asociarse con un mejor ajuste, y que el afrontamiento centrado en la emoción se asociaron con un ajuste psicológico más deficiente. Sin embargo, estos hallazgos generales no fueron concluyentes y la calidad del ajuste pareció estar relacionada con la naturaleza del estresor.

El afrontamiento es un proceso multidimensional complejo que es sensible a las demandas y recursos del ambiente, a las disposiciones de personalidad que influyen en la valoración de los factores estresantes y en la valoración de los recursos disponibles del individuo (Folkman y Moskowitz, 2004).

En el campo de la investigación de afrontamiento hay falta de consenso sobre la operacionalización del constructo "coping", y sobre las categorías de afrontamiento que mejor discriminan entre los niños (Compas et al., 2001). Teniendo en cuenta la complejidad del proceso de afrontamiento, un marco teórico podría proporcionar una estructura para integrar el vasto cuerpo de investigación. Pueden encontrarse propuestas como la de Skinner, Edge, Altman y Sherwood (2003) o el modelo 
propuesto y trabajado por el grupo de Frydenberg y Lewis $(1993,1997)$ que distingue tres categorías de estilos de afrontamiento: 1) Afrontamiento centrado en el problema; 2) en relación a los demás; y 3) improductivo (Freydenberg y Lewis, 1993, 1997). El primero de ellos, el afrontamiento dirigido a solucionar el problema, implica estrategias como las siguientes: resolución del problema, esfuerzo. El segundo, Afrontamiento basado en la relación con los demás: búsqueda de apoyo social, búsqueda de pertenencia, recurrir a amigos íntimos; y el tercero, el Afrontamiento improductivo son estrategias que no llevan a la solución del problema como, por ejemplo, las siguientes: hacerse ilusiones, no-afrontamiento, reducción de la tensión, ignorar el problema y preocupación. La tipología de estrategias improductivas también podría caracterizarse como disfuncional ya que ponen de manifiesto estos autores que estas últimas, formadas por estrategias emocionales, representan la forma menos eficaz de hacer frente a un problema.

Por todo lo anterior, la evaluación de las estrategias de afrontamiento empleadas por los estudiantes universitarios se considera fundamental ya que tiene implicaciones relevantes desde el punto de vista educativo y clínico ya que el empleo de estrategias funcionales o productivas guarda relación con mayor bienestar, calidad de vida, adaptación o ajuste en el ámbito universitario. Ello permitirá generar toma de conciencia sobre las estrategias empleadas por el estudiantado para que por parte del profesorado se desarrollen programas para el entrenamiento de aquellas consideradas más productivas ante los continuos retos y desafíos que se vienen planteando desde la Educación Superior.

\section{OBJETIVOS DE LA INVESTIGACIÓN}

Evaluar las estrategias de afrontamiento empleadas por una muestra de estudiantes universitarios españoles.

\section{MUESTRA Y/O PARTICIPANTES}

Los participantes fueron 169 estudiantes de grado pertenecientes a la Facultad de Ciencias de la Educación. Para el presente trabajo se ha seleccionado una submuestra final de 97 estudiantes que pertenecen a Primer Curso. Se han eliminado los datos de aquellas personas que no han completado todos y cada uno de los ítems del cuestionario o que eran estudiantes a tiempo parcial.

\section{METODOLOGÍA Y/O INSTRUMENTOS UTILIZADOS}

Para evaluar las estrategias de afrontamiento se aplicó la forma general de una escala validada en población española. Concretamente se ha aplicado el instrumento Escalas de Afrontamiento para Adolescentes ACS (Frydenberg y Lewis, 1996; Adaptación Española de Pereña y Seisdedos, TEA Ediciones, S. A.) que está formada por 80 elementos, uno abierto y 79 cerrados cuya escala de puntuación presenta cinco puntos: 1 . No me ocurre nunca o no lo hago y 5 . Me ocurre 0 lo hago con mucha frecuencia. En Se aplicó la Forma General, en la que se presentan una serie de 18 frases diferentes cuyo contenido se refiere a un total de 18 estrategias con las que el sujeto suele hacer frente a preocupaciones, dificultades o problemas sobre temas diferentes como las noas en la escuela, la relación con los amigos, la familia. Dichas estrategias corresponden a tres estilos de afrontamiento: afrontamiento improductivo, en relación con los demás y dirigido a la resolución del problema.

Los cuestionarios se aplicaron de forma colectiva en las distintas clases. Para la cumplimentación de los cuestionarios se aseguró la confidencialidad de la información y se informó a los participantes sobre la privacidad, y el carácter voluntario de la participación. 


\section{ESTRATEGIAS DE AFRONTAMIENTO EN UNA MUESTRA DE ESTUDIANTES UNIVERSITARIOS}

\section{RESULTADOS ALCANZADOS}

Este estudio presenta algunos de los datos preliminares obtenidos en esta investigación, analizados y discutidos según la literatura científica revisada, en la que se pone de manifiesto las estrategias de afrontamiento más empleadas por esta muestra de estudiantes universitarios, entre las que se encuentra tanto el empleo de estrategias de afrontamiento centradas en la solución del problema, así como aquellas otras centradas en la emoción.

A continuación se presenta la prevalencia de las estrategias más empleadas por la muestra de estudiantes universitarios en la Tabla 1.

Tabla 1. Estrategias de afrontamiento en estudiantes de grado

\begin{tabular}{|c|c|c|}
\hline Estrategia de afrontamiento & Media & Desviación típica \\
\hline 1. Buscar apoyo social & 18.6 & 3.5 \\
\hline $\begin{array}{l}\text { 2. Concentrarse en } \\
\text { resolver el problema }\end{array}$ & 19.2 & 2.4 \\
\hline $\begin{array}{l}\text { 3. Esforzarse y tener } \\
\text { éxito }\end{array}$ & 18.6 & 2.8 \\
\hline 4. Preocuparse & 20.3 & 3.43 \\
\hline $\begin{array}{l}\text { 5. Invertir en amigos } \\
\text { intimos }\end{array}$ & 17.2 & 3.1 \\
\hline 6. Buscar pertenencia & 17.6 & 3.0 \\
\hline 7. Hacerse ilusiones & 14.9 & 3.4 \\
\hline $\begin{array}{l}\text { 8. Falta de } \\
\text { afrontamiento }\end{array}$ & 9.4 & 2.9 \\
\hline $\begin{array}{l}\text { 9. Reducción de la } \\
\text { tensión }\end{array}$ & 11.0 & 3.3 \\
\hline 10. Acción social & 7.6 & 2.2 \\
\hline 11. Ignorar el problema & 6.8 & 2.3 \\
\hline 12. Autoinculparse & 11.7 & 3.7 \\
\hline 13. Reservarlo para sí & 10.6 & 3.0 \\
\hline $\begin{array}{l}\text { 14. Buscar apoyo } \\
\text { espiritual }\end{array}$ & 8.5 & 2.6 \\
\hline 15. Fijarse en lo positivo & 14.0 & 3.1 \\
\hline $\begin{array}{l}\text { 16. Buscar ayuda } \\
\text { profesional }\end{array}$ & 12.0 & 3.3 \\
\hline $\begin{array}{l}\text { 17. Buscar diversiones } \\
\text { relajantes }\end{array}$ & 10.2 & 2.0 \\
\hline 18. Distracción fisica & 9.0 & 2.6 \\
\hline
\end{tabular}

\section{DISCUSIÓN}

El objetivo del presente estudio fue examinar las estrategias de afrontamiento empleadas por una muestra de estudiantes universitarios españoles pertenecientes a Primer Curso; utilizando un diseño ex post facto.

El modelo teórico seguido en el presente trabajo es el propuesto por el grupo de Frydenberg y Lewis (1997) en el que se exhiben tres categorías de estilos de afrontamiento basado en la relación con los demás, diriguido a solucionar el problema e improductivo.

Puede señalarse, que con los continuos cambios que con el proceso de convergencia europea se viene produciendo en el ámbito universitario existen situaciones generadoras de estrés o preocupación. Por ejemplo, en un estudio reciente (Saleh, Camart y Romo, 2017) se pone de manifiesto como la entrada o primera toma de contacto en el ámbito universitario, como es el caso de la muestra que participa en este estudio que pertenece a primer curso, puede generar estrés y/o preocupación. De hecho, en este trabajo una de las puntuaciones medias más elevadas para la mues- 
tra participante es en la estrategia de afrontamiento denominada "Preocupación"; lo que nos lleva a la necesidad de plantear un programa de afrontamiento centrado entre otros aspectos, en las emociones que ayude una gestión lo más eficaz posible de las mismas.

Se ha localizado algún estudio previo (Scafarelli y García-Pérez, 2010) en el que también se aplica la escala empleada en este estudio a estudiantes uruguayos de primer curso por lo que puede servir de referencia en ese sentido, en el que se encontraron diferencias estadísticamente significativas en el empleo de estrategias de afrontamiento tales como "Buscar apoyo social" en función de la Carrera y del sexo. Concretamente los resultados demostraron en dicho estudio que los estudiantes de Ingeniería utilizan menos la estrategia "Buscar apoyo social" en comparación con los de Educación Inicial. Asimismo encontraron que las mujeres emplean más dicha estrategia apoyo social y otras como "Preocuparse" en comparación con los hombres.

\section{CONCLUSIONES}

Los datos obtenidos son relevantes para generar toma de conciencia de las estrategias de afrontamiento empleadas en el ámbito universitario para el diseño de un futuro programa o intervención psicoeducativa que permita entrenar aquellas estrategias de afrontamiento que resulten más eficaces para el afrontamiento de situaciones académicas o de la vida cotidiana generadoras de estrés. En dichos programas de entrenamiento de estrategias eficaces de afrontamiento del estrés cotidiano en futuros estudios importante la evaluación de las estrategias empleadas por los estudiantes en nuestro contexto además de considerarar el efecto de variables como la edad y el género.

\section{REFERENCIAS BIBLIOGRÁFICAS}

Compas, B. E., Connor-Smith, J. K., Saltzman, H., Thomsen, A. H., y Wadsworth, M. E. (2001). Coping with stress during childhood and adolescence: Problems, progress, and potential in theory and research. Psychological Bulletin, 127, 87-127.

Connor-Smith, J. K., y Compas, B. E. (2002). Vulnerability to social stress: Coping as a mediator or moderator of sociotropy and symptoms of anxiety and depression. Cognitive Therapy and Research, 26, 39-55.

Folkman, S., y Moskowitz, J. T. (2004). Coping: Pitfalls and promise. Annual Review Psychology, 55, 745-774.

Frydenberg, E. y Lewis, R. (1993). Boys play sport and girls turn to others: Age gender and ethnicity as determinants of coping. Journal of Adolescence, 16, 253-266.

Frydenberg, E. y Lewis, R. (1996). Escala de afrontamiento para adolescentes. Madrid: TEA.

Frydenberg, E. y Lewis, R. (1997). Escalas de afrontamiento para adolescentes. Adaptación española de Jaime Pereña y Nicolás Seisdedos. Madrid, TEA Ediciones.

Lazarus, R.S. (1991). Emotion and adaptation. In Pervin, L. A. (Ed.). Handbook of personality: Theory and research, (pp. 609-637), New York: Guilford.

Lazarus, R. S. y Folkman, R. S. (1984). Stress appraisal and coping. New York: Springer Publishing Company (traducción española, Estrés y procesos cognitivos, Barcelona, Martínez Roca, 1986).

Saleh, D., Camart, N. y Romo, L. (2017). Predictors of stress in Collegue Students. Frontiers in Psychology, 8(19), 1-8. Doi: 10.3389/fpsyg.2017.00019

Scafarelli, L. M. y García-Pérez, R. C. (2010). Estrategias de afrontamiento al estrés en una muestra de jóvenes universitarios uruguayos. Ciencias Psicológicas, IV(2), 165-175.

Skinner, Edge, Altman y Sherwood (2003). Searching for the structure of coping: A review and critique of category systems for classifying ways of coping. Psychological Bulletin, 129, 216-269. 
